\title{
Use of white rot fungi to improve the feed value of rice straw
}

\section{J W Cone ${ }^{1}$, J J P Baars ${ }^{2}$, A S M Sonnenberg ${ }^{2}$}

${ }^{1}$ Animal Nutrition Group, Wageningen University, Wageningen, Netherlands, ${ }^{2}$ Plant Sciences Group, Wageningen, Netherlands

Email:john.cone@wur.nl

Introduction In many countries straw is a main feed for cattle in the winter or in the dry season. However, the feed value of straw is too low to obtain high production levels. One of the main reason for the low feed value of straw is the high lignin level (for rice straw up to $5 \%$ ), preventing microbes to access the valuable carbohydrates and causing a low feed intake. In the past many attempts have been published to increase the feed value, using sodium hydroxide, urea and ammonia. Rodrigues et al. (2008) showed that enzymes of fungi are capable of increasing the digestibility of straw in rumen fluid. The aim of the present research was in investigate if growing fungi can be used to unseal the straw and to increase its feed value. Rice straw samples were incubated for different periods with two strains of white rot fungi before being fermented in rumen fluid, using the gas production technique.

Material and methods Rice straw from Thailand was chopped to pieces of $3 \mathrm{~cm}$. The white rot fungi Pleurotus ostreatus and Volvariella volvacea were inoculated for 6 days on $2 \%$ malt agar disks (diameter, $7-8 \mathrm{~mm}$ ) at $24^{\circ} \mathrm{C}$. Each 20 grams of chopped rice straw samples were put into $1000 \mathrm{ml}$ Erlenmeyer flasks with $80 \mathrm{ml}$ of distilled water. The flasks were autoclaved at $121{ }^{\circ} \mathrm{C}$ for $30 \mathrm{~min}$ and, after cooling, three agar disks of each fungus culture were added aseptically and incubated at $24{ }^{\circ} \mathrm{C}$ for 0 to 35 days. Three replications for each fungus culture of each incubation period were used. After incubation the rice straw samples were freeze dried, ground to $1 \mathrm{~mm}$ and analysed for fermentability in rumen fluid. Gas production incubations were performed as described by Cone et al. (1996), using rumen fluid from 2 non lactating Holstein Friesian cows receiving $1 \mathrm{~kg}$ of concentrate and ad libitum grass silage.
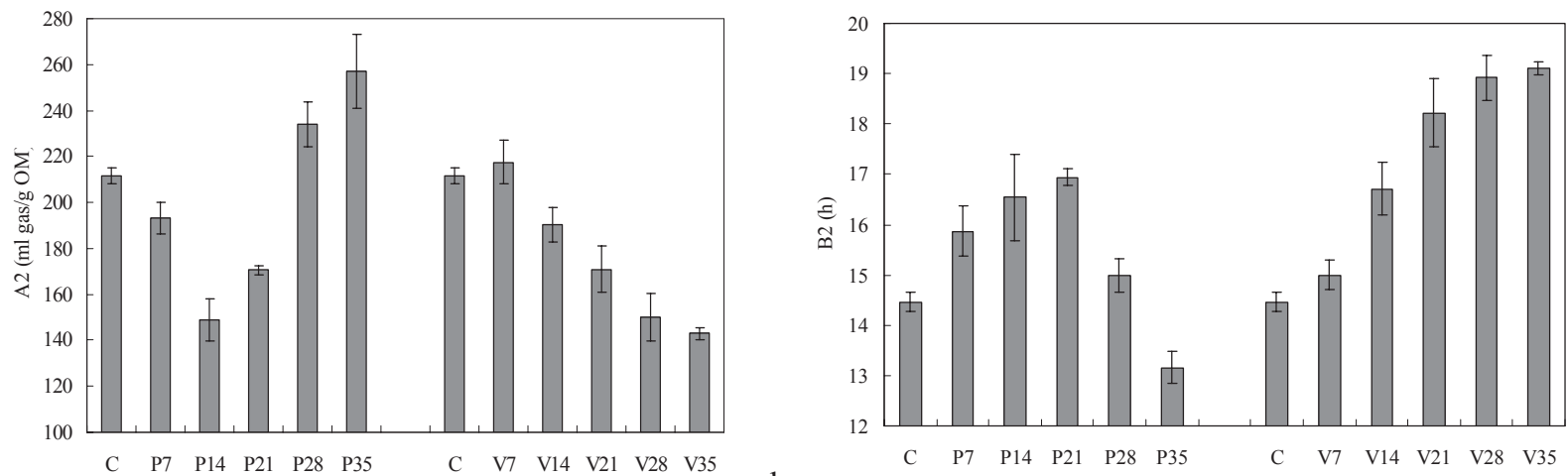

a

Figure 1 (a) Gas production (A2, ml gas/g OM) of the non-soluble fraction and (b) time to reach half of A2 (B2, h) of untreated rice straw $(\mathrm{C})$ and straw incubated for 7 to 35 days with the white rot fungi Pleurotis ostreatis (P) and Volvariella volvacea $(\mathrm{V})$.

Results Figure 1a shows the gas production ( $\mathrm{ml}$ gas/g OM), caused by fermentation of the non-soluble fraction (A2) of the straw samples after incubation with the white rot fungi $P$. ostreatus and $V$. volvacea for different periods (days). Figure $1 \mathrm{~b}$ shows the half time to reach A2 (B2), which is an indication of the rate of fermentation of the non-soluble fraction, in this case NDF. A lower value of B2 means an increased rate of fermentation. The results show that prolonged incubation with $V$. volvacea results in a lower fermentation of the NDF (decreased A2) and a slower rate of fermentation (increased B2), indicating that during the incubation of the straw with the fungus, $V$. volvacea used the most easily digestible parts of the straw, remaining less for the rumen microbes. The same was seen for straw samples incubated with P. ostreatus up to 14 days. Incubating the straw for a prolonged period with this fungus resulted in an increased fermentation of the NDF in rumen fluid with an increased rate. This indicates that initially $P$. ostreatus also used easy assessable carbohydrates, but after 2 weeks the fungus used the difficult degradable components, most likely lignin, increasing the degradability of the straw in rumen fluid.

Conclusions The results show that a prolonged incubation of rice straw with the white rot fungus $P$. ostreatus increases its feed value for ruminants.

\section{References}

Cone, J.W., Van Gelder, A.H., Visscher, G.J.W. and Oudshoorn, L. 1996. Animal Feed Science and Technology 61, 113128.

Rodrigues, M.A.M., Pinto, P., Bezerra, R.M.F., Dias, A.A., Guedes, C.V.M., Cardoso, V.M.G., Cone, J.W., Ferreira, L.M.M., Colaço, J., Sequeira, C.A. (2008) Animal Feed Science and Technology 141, 326-338. 